\title{
The AU-rich element mRNA decay-promoting activity of BRF1 is regulated by mitogen-activated protein kinase-activated protein kinase 2
}

\author{
SUSHMIT MAITRA, ${ }^{1,4}$ CHU-FANG CHOU, ${ }^{1,4}$ CHRISTIAN A. LUBER, ${ }^{2}$ KYUNG-YEOL LEE, $^{3}$ MATTHIAS MANN, ${ }^{2}$ \\ and CHING-YI CHEN ${ }^{1}$ \\ ${ }^{1}$ Department of Biochemistry and Molecular Genetics, University of Alabama at Birmingham, Birmingham, Alabama 35294, USA \\ ${ }^{2}$ Department of Proteomics and Signal Transduction, Max-Planck Institute of Biochemistry, D-82152 Martinsried, Germany \\ ${ }^{3}$ Department of Microbiology, School of Dentistry, Chonbuk National University, Chonbuk, South Korea 561-756
}

\begin{abstract}
Regulated mRNA decay is a highly important process for the tight control of gene expression. Inherently unstable mRNAs contain AU-rich elements (AREs) in the $3^{\prime}$ untranslated regions that direct rapid mRNA decay by interaction with decay-promoting AREbinding proteins (ARE-BPs). The decay of ARE-containing mRNAs is regulated by signaling pathways that are believed to directly target ARE-BPs. Here, we show that BRF1 involved in ARE-mediated mRNA decay (AMD) is phosphorylated by MAPK-activated protein kinase 2 (MK2). In vitro kinase assays using different BRF1 fragments suggest that MK2 phosphorylates BRF1 at four distinct sites, S54, S92, S203, and an unidentified site at the $C$ terminus. Coexpression of an active form of MK2 inhibits ARE mRNA decay activity of BRF1. MK2-mediated inhibition of BRF1 requires phosphorylation at S54, S92, and S203. Phosphorylation of BRF1 by MK2 does not appear to alter its ability to interact with AREs or to associate with mRNA decay enzymes. Thus, MK2 inhibits BRF1-dependent AMD through direct phosphorylation. Although the mechanism underlying this inhibition is still unclear, it appears to target BRF1-dependent AMD at a level downstream from RNA binding and the recruitment of mRNA decay enzymes.
\end{abstract}

Keywords: AU-rich elements (AREs); ARE-binding proteins; ARE-mediated mRNA decay; BRF1; MK2

\section{INTRODUCTION}

Regulation of mRNA decay plays a key regulatory role in gene expression (Wilusz et al. 2001; Wilusz and Wilusz 2004; Garneau et al. 2007). mRNAs encoding several cytokines and growth factors are degraded rapidly in order to minimize potentially inflammatory or oncogenic effects that may result from their overexpression. Many of these transcripts contain cis-acting instability elements within their $3^{\prime}$ untranslated regions that are thought to activate mRNA decay pathways (Wilusz et al. 2001). The AU-rich elements (AREs) appear to be prominent instability elements that direct rapid mRNA decay by a process referred to as ARE-mediated mRNA decay (AMD) (Chen and Shyu 1995; Bakheet et al. 2006).

\footnotetext{
${ }^{4}$ These authors contributed equally to this work.

Reprint requests to: Ching-Yi Chen, Department of Biochemistry and Molecular Genetics, University of Alabama at Birmingham, Birmingham, AL 35294, USA; e-mail: cchen@uab.edu; fax: (205) 975-2188.

Article published online ahead of print. Article and publication date are at http://www.rnajournal.org/cgi/doi/10.1261/rna.983708.
}

AMD is regulated by several RNA-binding proteins collectively called ARE-binding proteins (ARE-BPs). Destabilizing ARE-BPs bind ARE-containing mRNAs and target them for decay. The zinc finger proteins tristetraprolin (TTP) and butyrate response factor 1 and 2 (BRF1 and BRF2) are potent stimulators of AMD (Lai et al. 1999, 2000; Blackshear 2002; Stoecklin et al. 2002). TTP plays a critical role in the clearance of TNF $\alpha$ mRNA. Mice deficient in TTP exhibit a severe inflammatory phenotype due to overexpression of $\mathrm{TNF} \alpha$ resulting from decreased mRNA decay (Carballo et al. 1998). In addition, K homology splicing regulatory protein (KSRP) and AU-binding factor 1 (AUF1) are also destabilizing ARE-BPs (Zhang et al. 1993; Chen et al. 2001; Sarkar et al. 2003; Gherzi et al. 2004). The currently accepted model for AMD is that decay-promoting ARE-BPs recruit the mRNA decay machinery onto the mRNA molecule, thereby triggering its deadenylation, 5' decapping, and subsequent degradation (Gherzi et al. 2004; Lykke-Andersen and Wagner 2005; Chou et al. 2006).

Several signaling pathways, including the p38 MAPK and $\mathrm{PKB} /$ Akt pathways, regulate AMD (Winzen et al. 1999; Lasa et al. 2000; Dean et al. 2004; Schmidlin et al. 2004). p38 and its 
downstream kinase, MAPK-activated protein kinase 2 (MK2), appear to play a major role in the regulation of AMD. Stimulation of cells with IL-1 induces stabilization of the ARE-containing mRNA encoding COX-2, which is blocked by a p38 inhibitor (Ridley et al. 1998). Expression of constitutively active MKK6, an upstream activator of p38 or an active form of MK2, results in inhibition of AMD (Winzen et al. 1999; Lasa et al. 2000). Expression of a dominant-negative form of MK2 abolishes p38-induced stabilization of AREcontaining mRNAs (Winzen et al. 1999; Lasa et al. 2000). These findings demonstrate that MK2 plays a pivotal role in the inhibition of AMD by the p38 signaling pathway.

Although the exact mechanism by which p38 and PKB signaling pathways regulate AMD is still unclear, decaypromoting ARE-BPs are likely targets for protein kinases. MK2 phosphorylates TTP at Ser52 and Ser178, which results in inhibition of TTP-dependent AMD (Stoecklin et al. 2004). Phosphorylation of BRF1 by PKB at Ser90/ Ser92 and Ser203 impairs its decay-promoting activity (Schmidlin et al. 2004; Benjamin et al. 2006). Phosphorylation of both TTP and BRF1 at these serine residues results in an increased association with the 14-3-3 protein family, which is thought to exert an inhibitory effect on ARE mRNA decay activities of TTP and BRF1 (Chrestensen et al. 2004; Schmidlin et al. 2004; Stoecklin et al. 2004; Benjamin et al. 2006). Phosphorylation of KSRP by p38 inhibits its RNA binding activity, which appears to be required for stabilization of ARE-containing mRNAs encoding myogenin and p21, thereby regulating myogenesis (Briata et al. 2005). Furthermore, activation of PKB is required for stabilization of $\beta$-catenin mRNA, which is dependent on PKBmediated phosphorylation of KSRP (Gherzi et al. 2006).

In the present study, we show that MK2 phosphorylates BRF1 in vitro and identify three MK2 phosphorylation sites: Ser54, Ser92, and Ser203. Coexpression of an active form of MK2 inhibits BRF1 function in AMD. We demonstrate that Ser54, Ser92, and Ser203 are required for MK2-mediated inhibition, suggesting that MK2 inhibits BRF1 through direct phosphorylation. However, phosphorylation of BRF1 by MK2 does not appear to impair its ability to bind AREs or associate with mRNA decay enzymes. These results provide further evidence that decaypromoting ARE-BPs are direct targets for inhibition by signaling pathways that regulate AMD and suggest that the MK2-mediated inhibition of BRF1 function in mRNA decay likely occurs after the ARE binding and the recruitment of mRNA decay machinery.

\section{RESULTS}

\section{BRF1 is phosphorylated by MK2 in vitro}

To investigate whether BRF1 is a target of MK2, we performed in vitro kinase assays using recombinant BRF1 and TTP expressed as GST-tagged fusion proteins in
Escherichia coli. Purified proteins were incubated with activated recombinant MK2. We found that both GSTBRF1 and GST-TTP were phosphorylated by MK2 at a level comparable to HSP27, a known MK2 substrate (Fig. 1A). GST-BRF1 was also efficiently phosphorylated by endogenous MK2 immunoprecipitated from HEK 293 cells stimulated with anisomycin, a potent activator of the p38 signaling pathway (Fig. 1B). These results suggest that $\mathrm{BRF} 1$ is an MK2 substrate.

\section{Identification of in vitro MK2 phosphorylation sites within BRF1}

To determine MK2 phosphorylation sites, we expressed four BRF1 fragments as GST fusion polypeptides designated as F1-F4 (Fig. 2A). All four fragments were phosphorylated by MK2 in vitro (Fig. 2B). MK2 has been shown to preferentially phosphorylate the serine residue in the context of an RXXS consensus motif (Stokoe et al. 1993; Manke et al. 2005). Inspection of amino acid sequences of the four fragments revealed that each one contains one MK2 consensus motif (Fig. 2A). We substituted the Ser residue within the RXXS motif of each fragment with Ala and performed kinase assays with mutant polypeptides. Substitution of Ser54 abrogated phosphorylation of the F1 fragment (amino acids 1-64) by MK2 (Fig. 2C). The F2 fragment (amino acids 65-185) contains two overlapping RXXS motifs (Fig. 2A), with either or both Ser90 and Ser92 serving as potential phosphorylation sites. While substitution of Ser90 with Ala had no effect, no phosphorylation

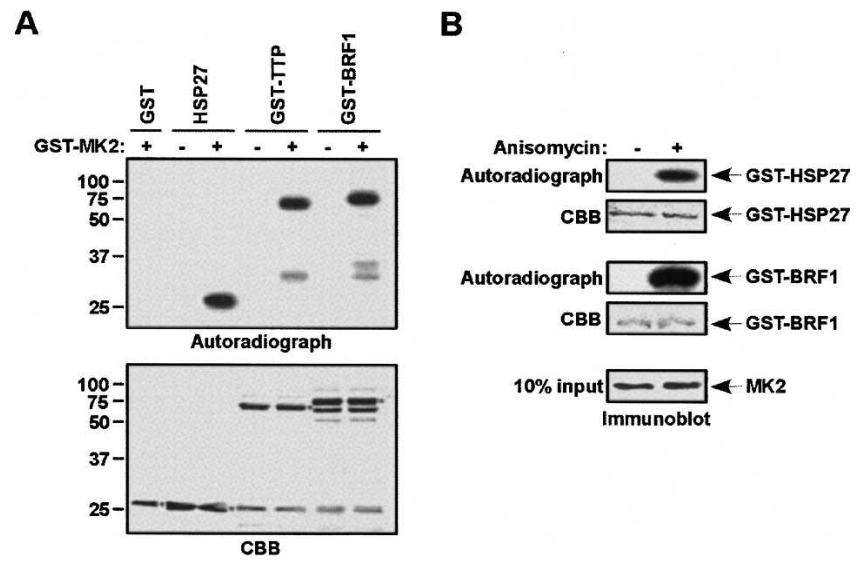

FIGURE 1. MK2 phosphorylates BRF1 in vitro. (A) His-HSP27, GST-TTP, and GST-BRF1 were incubated with or without an active MK2 in the presence of $\gamma^{-}{ }^{32} \mathrm{P}$-ATP for $40 \mathrm{~min}$ at $37^{\circ} \mathrm{C}$. Reactions were terminated by addition of SDS sample buffer, separated by SDSPAGE, and subjected to autoradiography (top panel). (Bottom panel) Coomassie brilliant blue (CBB) staining of the gel. (Asterisks) Target proteins. (B) 293 cells were treated with anisomycin $(200 \mathrm{ng} / \mathrm{mL})$ for $30 \mathrm{~min}$. Cell extracts were immunoprecipitated with an anti-MK2 antibody. The precipitates were subjected to kinase assays using GSTHSP27 or GST-BRF1 as substrates as described in A. Ten percent of the input used for immunoprecipitation was analyzed by anti-MK2 immunoblotting. 


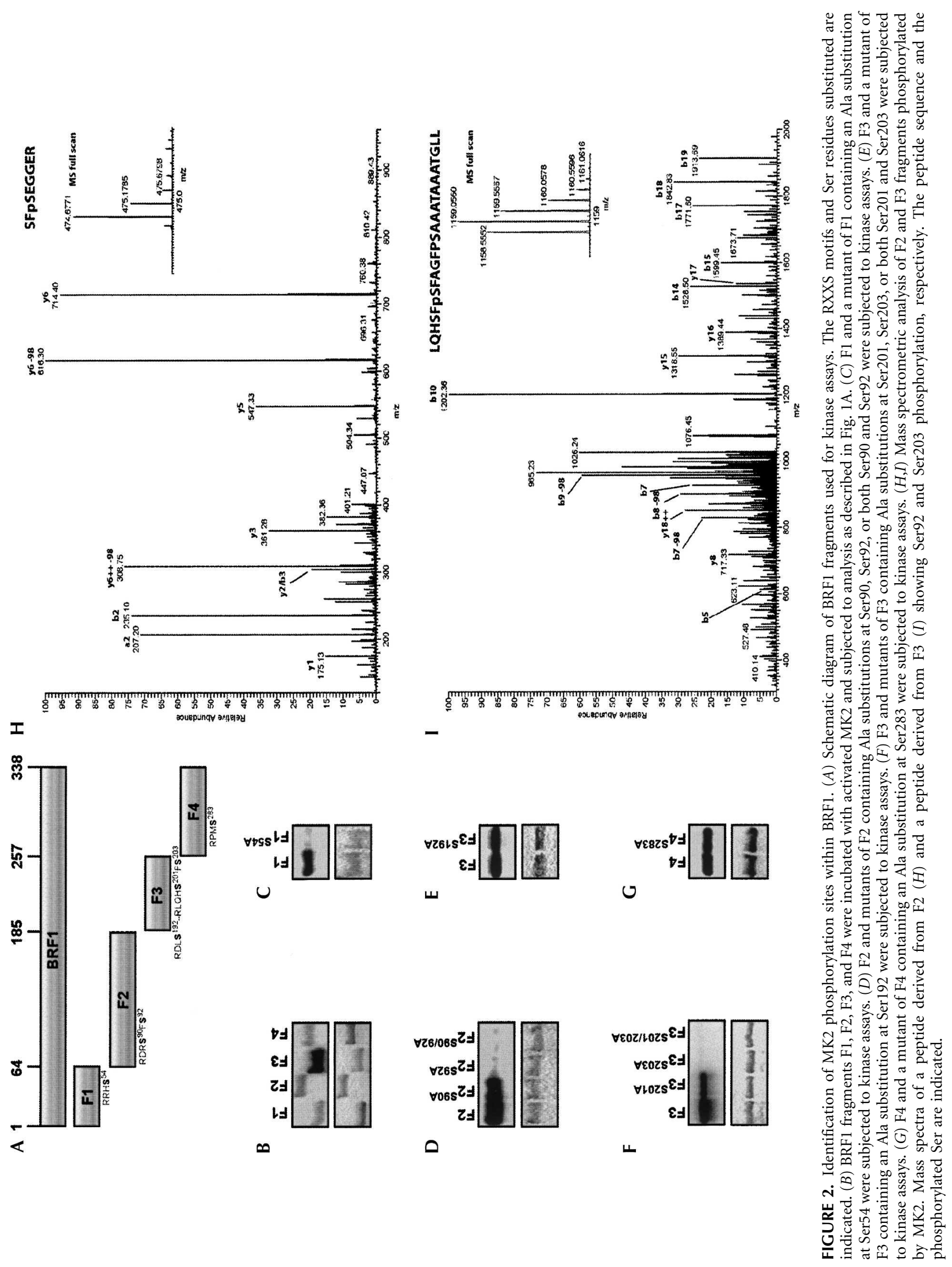


was detected in both S92A and S90/92A mutants, suggesting that Ser92 is the preferred MK2 phosphorylation site (Fig. 2D). Substitutions of Ser192 and Ser283 with Ala in the F3 fragment (amino acids 186-257) and F4 fragment (amino acids 257-338), respectively, did not significantly affect phosphorylation (Fig. 2E,G), suggesting that these Ser residues are not MK2 phosphorylation sites. Previous reports demonstrated that MK2 phosphorylates TTP at Ser178 and suggested that Ser203 of BRF1 is homologous to Ser178 in TTP (Benjamin et al. 2006). We generated Ala substitutions at Ser203 and the adjacent Ser201 in the F3 fragment. While substitution of Ser201 resulted in a moderate reduction in phosphorylation, substitution of Ser203 completely abrogated MK2-mediated phosphorylation of the F3 fragment (Fig. 2F), suggesting that Ser203 is critical for MK2-dependent phosphorylation.

To confirm that Ser92 and Ser203 are indeed phosphorylated by MK2, F2 and F3 fragments were phosphorylated by MK2 and subjected to mass spectrometric analysis. We identified a peptide derived from the F2 fragment containing a phosphate moiety at Ser92 (Fig. 2H) and a peptide derived from the F3 fragment with a phosphate group at Ser203 (Fig. 2I). We have recovered $\sim 95 \%$ of peptides; no other phosphorylation sites were identified (data not shown), and no peptides containing phosphate groups at either Ser90 or Ser201 were detected. It is therefore possible that substitution of Ser201 with Ala reduces the phosphorylation efficiency of the adjacent Ser203. The fact that the F3 fragment was more efficiently phosphorylated by MK2 than other fragments (Fig. 2B) is likely because it is a better substrate, but not because additional MK2 target sites are present in the fragment. Altogether, these results demonstrate that Ser92 and Ser203 are indeed phosphorylated by MK2. Furthermore, MK2 likely phosphorylates BRF1 at Ser54 and another unidentified site located within the F4 fragment.

\section{Coexpression of MK2 impairs the decay-promoting activity of BRF1}

To examine the effects of MK2-mediated phosphorylation on the function of BRF1 in AMD, we established a transcriptional pulse system in a cell line, SlowC, deficient in BRF1 (Stoecklin et al. 2002), and in its parental HT1080 fibrosarcoma cell line. In these cells, expression of a $\beta$ globin reporter mRNA containing the $3^{\prime}$ UTR of GMCSF (GB-ARE ${ }^{\mathrm{GMCSF}}$ ) is driven by a tetracycline-sensitive promoter, and addition of a tetracycline analog, doxycycline, blocks transcription of the mRNA. Thus, mRNA decay could be analyzed after its addition. GB-ARE ${ }^{\text {GMCSF }}$ mRNA was degraded in the parental HT1080 cell line with a halflife $\left(t_{1 / 2}\right)$ of $1.0 \mathrm{~h}$, while it was significantly stabilized in the SlowC cell line with a $t_{1 / 2}$ of $3.1 \mathrm{~h}$ (Fig. $3 \mathrm{~A}$ ). Expression of BRF1 in SlowC cells restored AMD (Fig. 3B). Coexpression of BRF1 with a constitutively active form of MK2 (MK2EE), in which both of the threonine residues phosphorylated

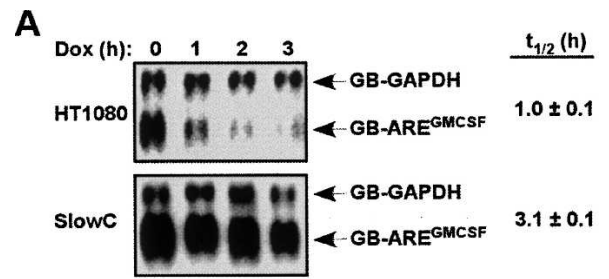

B
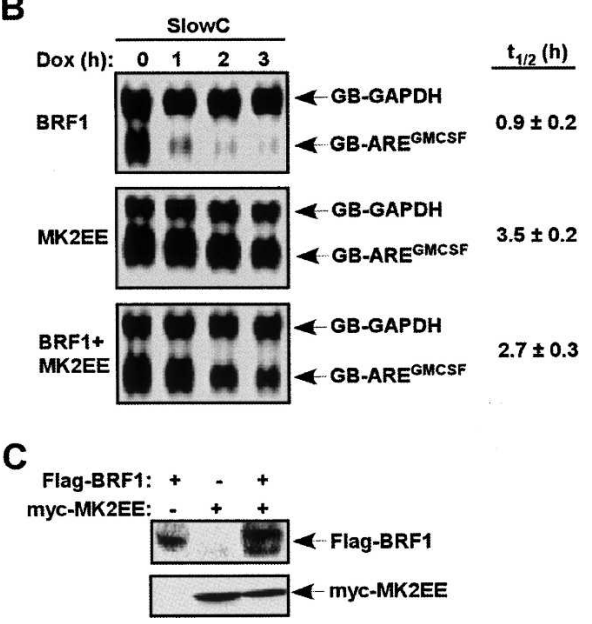

FIGURE 3. Coexpression of an active form of $\mathrm{MK} 2$ inhibits ARE mRNA decay activity of BRF1. (A) HT1080-TO or SlowC-TO cells were transfected with a construct expressing GB-ARE ${ }^{\mathrm{GMCSF}}$ mRNA under the control of a Tet-regulatory promoter and a construct constitutively expressing GB-GAPDH mRNA under the control of the CMV promoter. Total RNA was isolated at different time points after addition of doxycycline (Dox). The levels of GB-ARE ${ }^{\text {GMCSF }}$ and GBGAPDH mRNAs were analyzed by Northern blot. Signals of GB$\mathrm{ARE}^{\mathrm{GMCSF}}$ mRNA were quantitated by a phosphorimager and normalized to that of GB-GAPDH mRNA. The calculated half-lives $\left(t_{1 / 2} ; n=\right.$ 2) of GB-ARE ${ }^{\text {GMCSF }}$ mRNA are shown as mean values \pm standard deviations. (B) GB-ARE ${ }^{\text {GMCSF }}$ and GB-GAPDH mRNAs were expressed in SlowC-TO cells with constructs expressing Flag-BRF1, myc-MK2EE, or both. Total RNA was isolated at different time points after addition of doxycycline (Dox). The levels of GB-ARE ${ }^{\text {GMCSF }}$ and GBGAPDH mRNAs were analyzed as described in $A$. The calculated $t_{1 / 2}$ $(\mathrm{n}=2-3)$ of GB-ARE ${ }^{\mathrm{GMCSF}}$ mRNA are shown. $(C)$ Extracts from $B$ were analyzed by immunoblotting using anti-Flag or anti-myc antibodies.

and required for its activation by $\mathrm{p} 38$ are substituted with glutamate (Engel et al. 1995), decreased the BRF1-dependent decay of GB-ARE ${ }^{\text {GMCSF }}$ mRNA (Fig. 3B). These results indicate that MK2 impairs the function of BRF1 in AMD.

\section{MK2 can act on BRF1 through a PKB-independent manner}

It was demonstrated that $\mathrm{PKB}$ and MK2 form a complex together with HSP27, and MK2 phosphorylates $\mathrm{PKB}$ at Ser473, which results in its activation (Rane et al. 2001; Zheng et al. 2006; Wu et al. 2007). Since MK2 phosphorylates BRF1 at the sites (Ser92 and Ser203) shared by PKB, which also inhibits BRF1 function in AMD (Schmidlin et al. 2004; Benjamin et al. 2006), we examined whether PKB activity is necessary for MK2-mediated inhibition of BRF1. 
We first examined whether expression of MK2EE leads to activation of $\mathrm{PKB}$ in SlowC-TO cells. Coexpression of MK2EE moderately increased (about twofold) PKB phosphorylation (Fig. 4A). Transfection efficiency in SlowC cells was typically $30 \%$ when analyzed by expression of a GFP marker (data not shown). We next examined whether down-regulation of PKB expression by RNAi could interfere with the inhibition of BRF1 by MK2. While PKB expression was down-regulated by $50 \%-70 \%$ after two rounds of siRNA transfection (Fig. 4C), the ARE-mRNA decay activity of BRF1 was still inhibited by coexpression of MK2EE (Fig. 4B).

As the down-regulation of $\mathrm{PKB}$ by siRNA was $70 \%$ at most, the residual $\mathrm{PKB}$ activity in the transfected cells might still potentiate MK2-mediated inhibition of BRF1. We next examined whether BRF1-dependent AMD could be inhibited by anisomycin treatment, which activates both $\mathrm{PI} 3 \mathrm{~K} / \mathrm{PKB}$ and $\mathrm{p} 38 / \mathrm{MK} 2$ signaling pathways (Figs. 4D, 1B). Co-incubation of wortmannin, a PI3K inhibitor, but not SB203580, a p38 inhibitor, completely disrupted PKB acti-
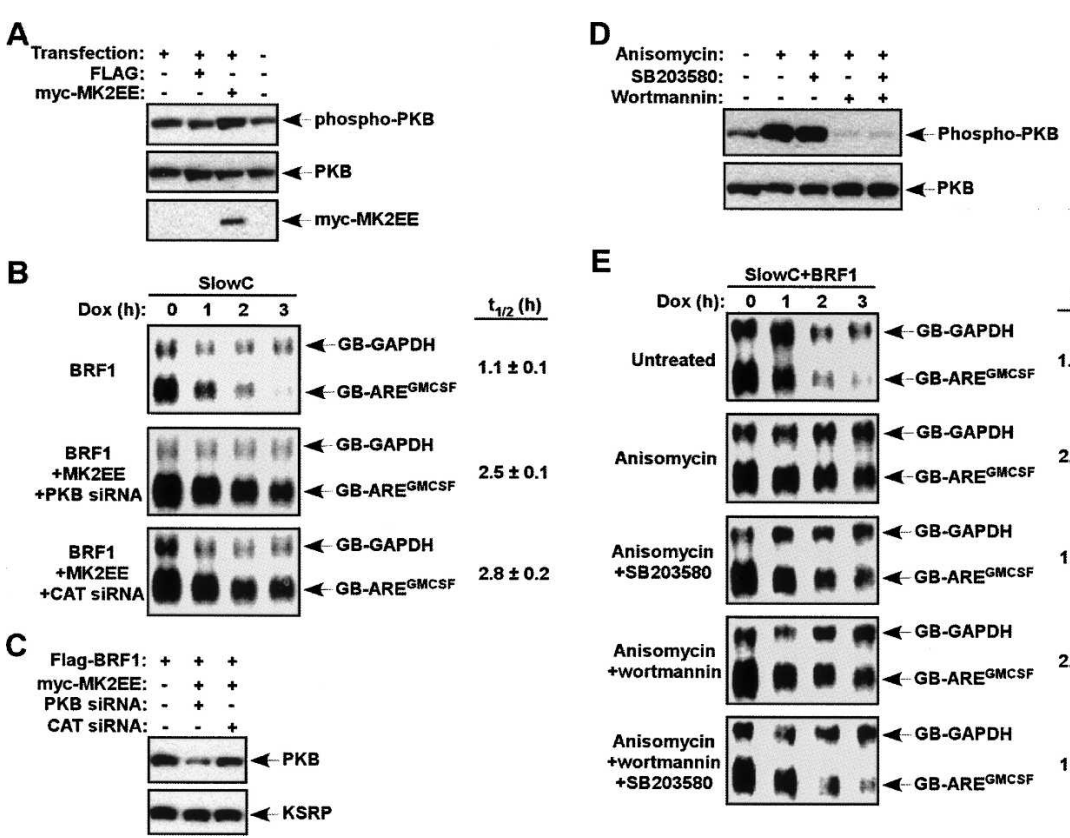

E

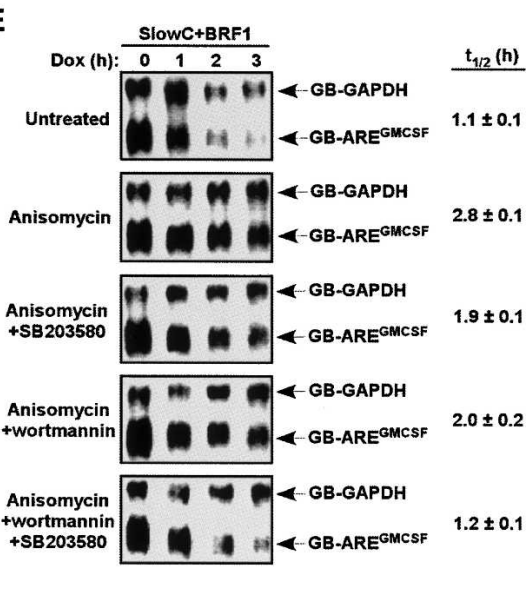

FIGURE 4. MK-2 mediated inhibition of BRF1 is independent on PKB activity. (A) SlowCTO cells were either left untransfected or were transfected without DNA or with plasmids expressing Flag or myc-MK2EE. Cell extracts were analyzed by anti-phospho-Ser ${ }^{473}$-PKB, anti$\mathrm{PKB}$, or anti-myc immunoblotting. (B) SlowC-TO cells were transfected with constructs expressing GB-ARE ${ }^{\mathrm{GMCSF}}$ and GB-GAPDH mRNAs and constructs expressing Flag-BRF1 and myc-MK2EE in the presence of a PKB siRNA or a control siRNA (CAT). The decay of GB$\mathrm{ARE}^{\mathrm{GMCSF}}$ mRNA was analyzed. The calculated $\mathrm{t}_{1 / 2}(\mathrm{n}=2)$ of GB-ARE ${ }^{\mathrm{GMCSF}}$ mRNA are shown. $(C)$ Down-regulation of PKB by siRNA was analyzed with extracts from $B$ by anti-PKB or anti-KSRP immunoblotting. (D) SlowC-TO cells were untreated or pretreated with DMSO, SB203580 (5 uM), wortmannin (200 nM), or both compounds for $30 \mathrm{~min}$ and further treated with anisomycin $(200 \mathrm{ng} / \mathrm{mL})$ for $30 \mathrm{~min}$. Cell extracts were analyzed by anti-phospho-PKB or anti-PKB immunoblotting. (E) GB-ARE ${ }^{\text {GMCSF }}$ and GB-GAPDH mRNAs were expressed in SlowC-TO cells with Flag-BRF1. Cells were left untreated or were treated with anisomycin in the presence of SB203580, wortmannin, or both. The decay of GB-ARE ${ }^{\mathrm{GMCSF}}$ mRNA was analyzed and the calculated $t_{1 / 2}(n=2)$ of GB-ARE ${ }^{\text {GMCSF }}$ mRNA are shown. vation by anisomycin (Fig. 4D). As shown in Figure 4E, While co-incubation with either wortmannin or SB203580 both compounds together with anisomycin substantially, if not completely, abrogated its ability to inhibit BRF1 function in AMD (Fig. 4E). As anisomycin still inhibits BRF1 in the absence of either PKB or MK2 activation and addition of SB203580 with wortmannin further decreased anisomycin-mediated inhibition, we suggest that activation of MK2 by anisomycin is able to inhibit BRF1-dependent AMD in absence of $\mathrm{PKB}$ activation. Altogether, these data (lation and that both $\mathrm{PKB}$ and $\mathrm{p} 38$ pathways act AMD.

\section{MK2-mediated inhibition of BRF1 is dependent on phosphorylation of Ser54, Ser92, and Ser203}

We next examined whether MK2-mediated phosphorylation is necessary for the inhibition of BRF1 function in AMD. We performed mRNA decay assays in SlowC cells to investigate the effect of MK2 on the decay-promoting activity of BRF1 mutants containing Ser to Ala substitutions. Expression of some of these BRF1 mutants restored AMD in SlowC cells, indicating that they are not functionally impaired in mRNA decay (Fig. 5A; data not shown). While MK2EE coexpression strongly compromised the decay-promoting activity of wild-type BRF1, the BRF1(S203A) mutant, but not the BRF1(S92A) mutant, significantly resisted MK2-mediated inhibition (Fig. 5B). A BRF1 mutant (S54/92/203A) containing additional Ala substitutions at both Ser54 and Ser92 in combination with the Ser203 mutation completely escaped MK2mediated inhibition (Fig. 5B). These results suggest that phosphorylation of Ser92 and Ser203, and likely Ser54, by MK2 is necessary for the inhibition of BRF1-dependent AMD.

\section{Phosphorylation of BRF1 by MK2 does not affect its RNA binding property or its ability to associate with mRNA decay enzymes}

To investigate the mechanism of inhibition of BRF1, we examined whether 
A

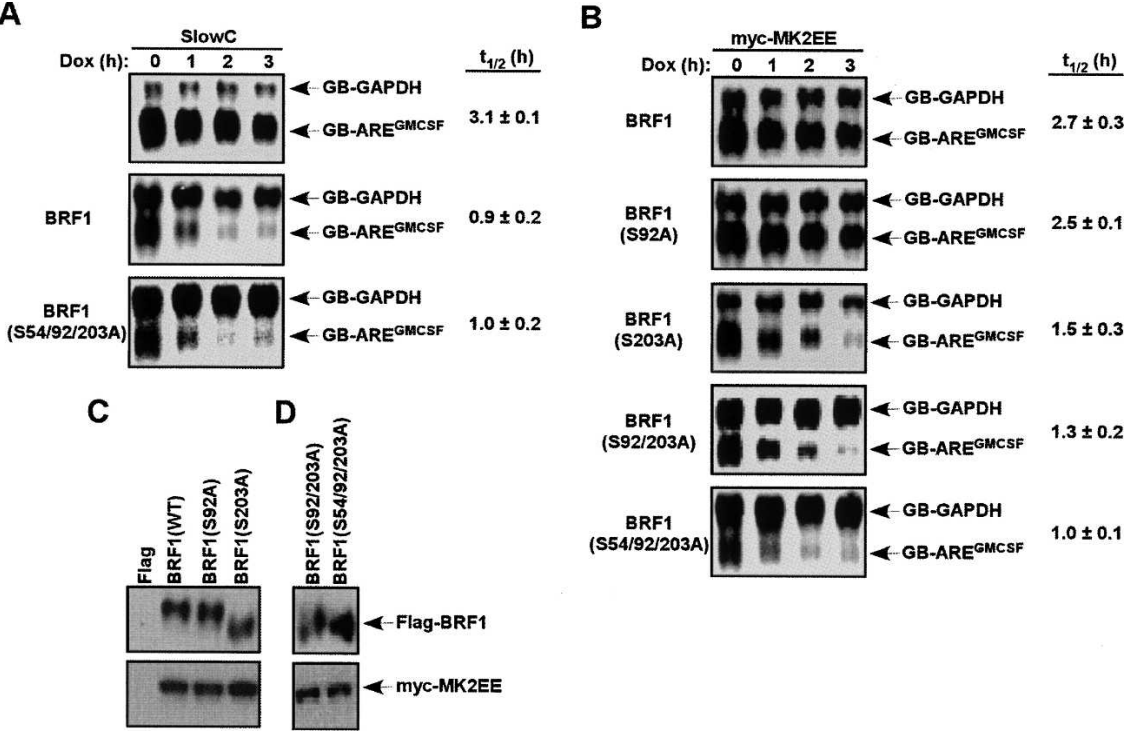

FIGURE 5. Inhibition of BRF1 function in AMD depends on MK2-mediated phosphorylation. (A) SlowC-TO cells were transfected with constructs expressing GB-ARE ${ }^{\mathrm{GMCSF}}$ and GBGAPDH mRNAs and constructs expressing different Flag-tagged BRF1 as indicated. The decay of GB-ARE ${ }^{\mathrm{GMCSF}}$ mRNA was analyzed. The calculated $\mathrm{t}_{1 / 2}(\mathrm{n}=2-3)$ of GB-ARE ${ }^{\mathrm{GMCSF}}$ mRNA are shown. (B) GB-ARE ${ }^{\mathrm{GMCSF}}$ and GB-GAPDH mRNAs were expressed in SlowC-TO cells in the presence of different Flag-tagged BRF1 mutants and myc-MK2EE. The decay of GB-ARE ${ }^{\text {GMCSF }}$ mRNA was analyzed. The calculated $t_{1 / 2}(n=2-3)$ of GB-ARE ${ }^{\text {GMCSF }}$ mRNA are shown. $(C, D)$ Expression of transfected Flag-BRF1 mutants and myc-MK2EE. Extracts from $B$ were either immunoprecipitated with anti-Flag and analyzed by immunoblotting using anti-BRF1 (top panels) or directly analyzed by anti-myc immunoblotting (bottom panels). Differential mobility of BRF1 observed in $C$ is likely due to phosphorylation of Ser203 by MK2.

phosphorylation of BRF1 by MK2 decreases its AREbinding activity. Flag-tagged BRF1 was expressed in 293 cells, since transfected BRF1 was poorly expressed in HT1080 or SlowC cells (Fig. 3C; data not shown), in the absence or presence of MK2EE. UV cross-linking experiments using ARE ${ }^{\mathrm{GMCSF}} \mathrm{RNA}$ or a non-ARE RNA as substrates were performed to induce formation of RNABRF1 complexes, which were immunoprecipitated with an anti-Flag antibody. The amounts of ARE RNA cross-linked to Flag-BRF1 were similar regardless of whether MK2EE was coexpressed or not (Fig. 6). No RNA/BRF1 complexes were detected using a non-ARE RNA (Fig. 6), suggesting that BRF1 specifically binds the ARE RNA.

We next examined the effect of MK2EE expression on the association of BRF1 with mRNA decay enzymes, including the deadenylase CCR4, the decapping enzyme DCP2, and the exosome component RRP4. HA-tagged CCR4 or myc-tagged DCP2 was coexpressed with FlagBRF1 in the absence or presence of MK2EE. While HACCR4, myc-DCP2, and endogenous RRP4 were coimmunoprecipitated with Flag-BRF1, no significant differences in the amounts of coimmunoprecipitated enzymes were detected when BRF1 was coexpressed with MK2EE (Fig. 7). Coexpression of MK2EE with BRF1 increased its association with 14-3-3 proteins (Fig. 7A), consistent with previous observations (Schmidlin et al. 2004; Stoecklin et al. 2004; Benjamin et al. 2006), suggesting that MK2EE is active in transfected 293 cells. The interaction of BRF1 with 14-3-3 in the absence of MK2EE (Fig. 7A, lane 2) was due to the basal activities of the PI3K/PKB and p38 signaling pathways, as treatment of cells with wortmannin or SB203580 disrupted the interaction (data not shown). In addition, the Flag-BRF1 mutant (S54/92/203A) failed to interact with $14-3-3$, but retained its ability to associate with mRNA decay enzymes (data not shown), suggesting that the interaction with $14-3-3$, but not the decay enzymes, is dependent on BRF1 phosphorylation. These results suggest that MK2-mediated inhibition of BRF1 function in AMD may not result from a decrease in RNA binding or impairment of its ability to recruit the tested mRNA decay enzymes.

\section{DISCUSSION}

Previous studies aimed at understanding the mechanisms by which signaling pathways stabilize ARE-containing mRNAs have led to identification of TTP and BRF1 as targets of MK2 and PKB, respectively (Schmidlin et al. 2004; Stoecklin et al. 2004; Benjamin et al. 2006). Phosphorylation of TTP by MK2 or BRF1 by PKB inhibits their ARE-mRNA decay functions. While MK2 was shown to phosphorylate BRF1 (Benjamin et al. 2006), its effect on the function of BRF1 in AMD has not been
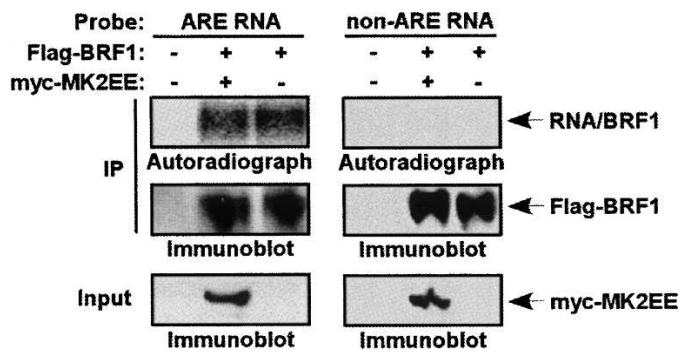

FIGURE 6. Coexpression of MK2 with BRF1 does not alter its ability to interact with an ARE. Flag-BRF1 was expressed in 293 cells in the absence or presence of myc-MK2EE. Cytoplasmic extracts were prepared and incubated with ${ }^{32} \mathrm{P}$-labeled $\mathrm{ARE}^{\mathrm{GMCSF}} \mathrm{RNA}$ or non-ARE E4 RNA (Gherzi et al. 2004), and UV cross-linking assays were performed. The UV cross-linking reactions were immunoprecipitated with anti-Flag and immunoprecipitates were analyzed by SDS-PAGE. The RNA-BRF1 complexes were detected by autoradiography (top panel). The immunoprecipitates were also analyzed by anti-Flag immunoblotting (middle panel). Ten percent of the input used for UV cross-linking assays was also analyzed by anti-myc immunoblotting (bottom panel). 


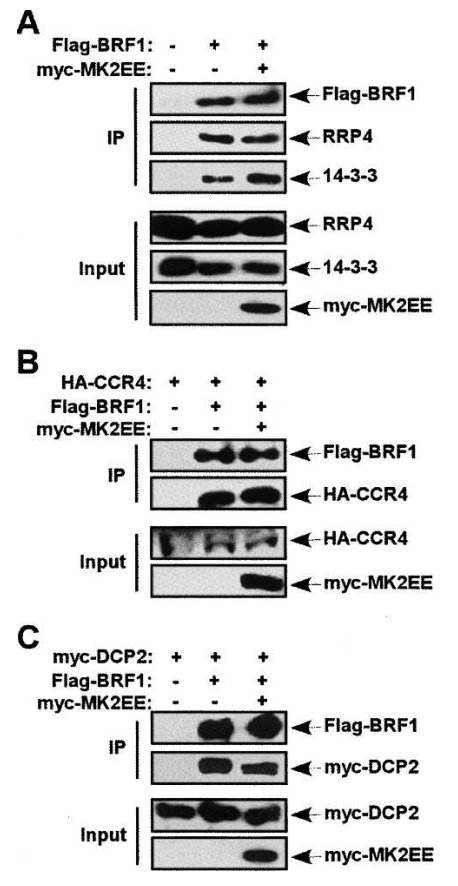

FIGURE 7. Coexpression of MK2 with BRF1 does not alter its ability to associate with mRNA decay enzymes. (A) Flag-BRF1 was expressed in 293 cells in the absence or presence of myc-MK2EE. RNase-treated cell extracts were subjected to anti-Flag immunoprecipitation. The precipitates were analyzed by anti-Flag, anti-RRP4, or anti-14-3-3 immunoblotting. Five percent of the input used for immunoprecipitation reactions was also analyzed by anti-RRP4, anti-14-3-3, or antimyc immunoblotting. (B) Flag-BRF1 and HA-CCR4 were expressed in 293 cells in the absence or presence of myc-MK2EE. RNase-treated cell extracts were subjected to anti-Flag immunoprecipitation. The precipitates were analyzed by anti-Flag or anti-HA immunoblotting. Five percent of the input used for immunoprecipitation reactions was also analyzed by anti-HA or anti-myc immunoblotting. (C) FlagBRF1 and myc-DCP2 were expressed in 293 cells in the absence or presence of myc-MK2EE. RNase-treated cell extracts were subjected to anti-Flag immunoprecipitation. The precipitates were analyzed by anti-Flag or anti-DCP2 immunoblotting. Five percent of the input used for immunoprecipitation reactions was also analyzed by antiDCP2 or anti-myc immunoblotting.

previously investigated. In this study, we show that MK2 inhibits the ARE-mRNA decay-promoting activity of BRF1. This inhibition is dependent on MK2-mediated phosphorylation of BRF1. Thus, our data and the previous studies (Schmidlin et al. 2004; Stoecklin et al. 2004; Briata et al. 2005; Benjamin et al. 2006; Gherzi et al. 2006) strongly suggest that decay-promoting ARE-BPs are targeted by signaling pathways through direct phosphorylation.

Using recombinant proteins expressed in E. coli, we found that BRF1 is phosphorylated by MK2 at a minimum of four distinct sites in vitro. Substitution of Ser54, Ser92, or Ser203 with Ala abrogates MK2-mediated phosphorylation of fragments containing each of these Ser residues, suggesting that they are MK2 target sites. Indeed, we confirmed that MK2 phosphorylates Ser92 and Ser203 by mass spectrometry. In addition, our results indicate that there may be one or more MK2 phosphorylation sites within the $\mathrm{C}$ terminus (amino acids 257-338). Currently, we do not know whether the identified sites are indeed phosphorylated by MK2 in vivo, and additional or fewer sites may be identified in vivo in the context of full-length BRF1. BRF1 was previously shown to be phosphorylated by MK2. In that study, the sites phosphorylated by MK2 were not identified; however, it was suggested that Ser203 is not an MK2 target site (Benjamin et al. 2006). Although we do not know the reason for the discrepancy between our phosphorylation assays and previous experiments performed by Benjamin et al., we speculate that the disparity may result from differences in the BRF1 fragments analyzed: amino acids 143-233 (Benjamin et al. 2006) versus amino acids 185-257 (our studies).

We investigated the functional significance of MK2mediated phosphorylation on BRF1-dependent AMD and demonstrated that MK2-mediated inhibition of BRF1 appears to rely on phosphorylation at Ser54, Ser92, and Ser203. Our results also suggest that mutations at the identified MK2 phosphorylation sites confer additive resistance to MK2-mediated inhibition. Recent reports by Moroni and colleagues have identified Ser90/Ser92 and Ser203 as PKB phosphorylation sites essential for PKBmediated inhibition of BRF1 function in AMD (Schmidlin et al. 2004; Benjamin et al. 2006). We further investigated whether MK2 can function independently on PKB activity, since previous studies demonstrated that MK2 phosphorylates and activates PKB (Rane et al. 2001; Zheng et al. 2006; Wu et al. 2007). We showed that down-regulation of $\mathrm{PKB}$ expression had little effect on MK2-mediated inhibition of BRF1. In addition, we also found that BRF1-dependent AMD was inhibited by treatment with anisomycin. The inhibition was released only by coincubation of cells with both wortmannin and SB203580, but not by co-incubation with either compound alone (Fig. 4E), suggesting that activation of either PI3K or the p38 signaling pathway is sufficient for BRF1 inhibition. Altogether, our results and previous results by the Moroni group strongly suggest that PKB and MK2 act independently on BRF1-dependent AMD through phosphorylation. However, it is likely that MK2 may also act on BRF1 through a PKB-dependent manner since coexpression of MK2EE increases PKB phosphorylation. Since MK2 and $\mathrm{PKB}$ share target sites, it is likely that both kinases employ similar mechanisms to block BRF1-dependent AMD.

While it is known that phosphorylation results in the inhibition of the mRNA decay-promoting properties of TTP and BRF1, the mechanisms involved remain nebulous. TTP and BRF1 bind to ARE-containing mRNAs and are thought to function by recruiting mRNA decay enzymes onto the mRNAs, consequently targeting them for degradation. We examined the effect of phosphorylation by MK2 on the abilities of BRF1 to recognize AREs and to associate 
with mRNA decay enzymes. We did not detect any perturbations in the abilities of BRF1 to bind AREs and to associate with any of the tested components of the mRNA decay machinery. Thus, our results do not support the hypothesis that MK2-mediated inhibition of BRF1dependent AMD results from a decrease in ARE-binding and mRNA decay enzyme association. However, we cannot exclude that there may be cell-type-specific effects, in which the RNA binding and mRNA decay enzyme recruitment by BRF1 is decreased, or that the recruitment of other untested mRNA decay enzymes is decreased upon MK2mediated phosphorylation. Alternatively, MK2 may interfere with the formation of a ternary complex containing ARE-RNA, BRF1, and mRNA decay enzymes, which is believed to be an active complex for mRNA decay. We hypothesize that additional critical factors may regulate the activity of the recruited mRNA decay machinery. These factors either can play an inhibitory role in mRNA decay, which are recruited upon MK2-mediated phosphorylation, or they might serve as activators of mRNA decay, which are no longer recruited upon MK2-mediated phosphorylation. Recent studies have implicated the 14-3-3 protein family in the regulation of ARE-mRNA decay activities of BRF1 and TTP (Stoecklin et al. 2004; Benjamin et al. 2006). However, it is unclear whether 14-3-3 proteins play a direct role in the inhibition of TTP and BRF1 by protein kinases.

Lykke-Andersen and Wagner previously reported that both the N-terminal (NTD) and C-terminal (CTD) domains of TTP and BRF1 possess mRNA decay-promoting activities (Lykke-Andersen and Wagner 2005). The NTD of TTP, but not the CTD, is responsible for the association with mRNA decay enzymes (Lykke-Andersen and Wagner 2005). Our results demonstrate that MK2 phosphorylates BRF1 at both the NTD and CTD, and this phosphorylation plays a critical role in the inhibition of BRF1-dependent AMD. The fact that that MK2 does not significantly disrupt BRF1 association with mRNA decay enzymes suggests that phosphorylation of the NTD by MK2 does not regulate the recruitment of mRNA decay enzymes. The CTD of TTP, and perhaps of BRF1, does not associate with any tested mRNA decay enzymes, but contains a second mRNA decay activation domain (Lykke-Andersen and Wagner 2005). Although the mechanism by which the CTDs of BRF1 and TTP activate mRNA decay pathways is still unknown, we hypothesize that unidentified factors critical for the control of the activity of recruited mRNA decay machinery are recruited or no longer recruited by the CTD of BRF1 upon phosphorylation of Ser203 by MK2. The exact mechanisms for MK2-dependent inhibition of BRF1 require further investigation, and the identification of additional factors that regulate the functions of BRF1 and TTP upon phosphorylation should help to elucidate the mechanism and regulation of ARE-mediated mRNA decay.

\section{MATERIALS AND METHODS}

\section{Plasmids}

mRNA reporter plasmids, pTRE-GB-ARE ${ }^{\mathrm{GMCSF}}$ and pcDNA-GBGAPDH, have been described previously (Chou et al. 2006; Lin et al. 2007). Flag-tagged BRF1 was constructed by subcloning the coding region of human BRF1, amplified by PCR, between the EcoRI and XhoI sites of pcDNA-FlagB (Lin et al. 2007). A BamHI/ $\mathrm{XbaI}$ fragment containing the coding region of DCP2 was subcloned into a pcDNA-myc vector to generate pcDNA-mycDCP2. Constructs expressing myc-MK2EE and HA-CCR4 were described previously (Engel et al. 1995; Yamashita et al. 2005). BRF1 and its fragments were amplified by PCR and subcloned into pGEX vectors (Amersham) to produce GST-tagged BRF1 and BRF1 fragments. Site-directed mutagenesis was carried out with the Stratagene QuikChange kit as per the manufacturer's protocol, and mutations were confirmed by DNA sequencing.

\section{Purification of recombinant proteins and activation of MK2}

GST- and His-tagged proteins were expressed in BL21 (DE3) cells and purified with glutathione-sepharose 4B (Amersham) or NiNTA agarose (Qiagen) as per the manufacturer's instructions. To activate MK2, GST-MK2 was immobilized on glutathione sepharose beads and incubated with constitutively active His-tagged MKK6 and His-tagged p38 in a kinase buffer $(20 \mathrm{mM}$ Hepes [pH 7.5], $10 \mathrm{mM} \mathrm{MgCl}_{2}$, $1 \mathrm{mM}$ DTT, $20 \mathrm{mM} \beta$-glycerol phosphate, $10 \mathrm{mM}$ PNPP, $50 \mu \mathrm{M}$ sodium vanadate, and $20 \mu \mathrm{M}$ ATP) for $40 \mathrm{~min}$ at $37^{\circ} \mathrm{C}$, after which the MK2-containing beads were washed five times with PBS. Activated GST-MK2 was eluted off the beads with elution buffer (50 mM Tris- $\mathrm{HCl}[\mathrm{pH} 8.0]$ and $5 \mathrm{mM}$ glutathione).

\section{In vitro kinase assays}

$0.5-1 \mu \mathrm{g}$ of recombinant proteins were incubated with $50 \mathrm{ng}$ of activated GST-MK2 and $\gamma-{ }^{32} \mathrm{P}$-ATP in kinase buffer for $40 \mathrm{~min}$ at $37^{\circ} \mathrm{C}$. To minimize background phosphorylation by any contaminated His-p38 during activation of MK2, kinase assays were carried out in the presence of $5 \mu \mathrm{M}$ SB203580. The reactions were denatured in SDS sample buffer and subjected to polyacrylamide gel electrophoresis. Gels were stained with coomassie brilliant blue and subjected to autoradiography.

\section{Antibodies}

Mouse monoclonal anti-Flag (M2), anti-myc (9E10), anti-HA (12CA5), anti-MK2, and anti-14-3-3 $\beta$ (H8), which cross-reacts several isoforms, were purchased from Sigma, Roche, Upstate Biotechnology, and Santa Cruz Biotechnology, respectively. Antiphospho-Ser ${ }^{473}$-PKB and anti-PKB antibodies were purchased from Cell Signaling. Anti-DCP2 (Wang et al. 2002), anti-RRP4 (Mitchell et al. 1997), and anti-BRF1 (Schmidlin et al. 2004) have been described previously.

\section{mRNA decay assays}

SlowC-Tet Off (TO) cells were established as previously described for HT1080-TO cells (Garcia-Mayoral et al. 2007). HT-TO or 
SlowC-TO cells were transfected using lipofectamine. After transfection, cells were incubated with tetracycline-free medium for $16 \mathrm{~h}$, followed by addition of doxycycline $(2 \mu \mathrm{g} / \mathrm{mL})$. Total RNA was isolated at different times, and reporter mRNA levels were analyzed by Northern blot as previously described (Chou et al. 2006).

\section{Extract preparation and immunoprecipitation assays}

Cytoplasmic extracts were prepared from transfected cells and subjected to RNase A treatment as described (Lykke-Andersen 2002). The RNase-treated extracts were incubated with $10 \mu \mathrm{L}$ (bed volume) of anti-Flag agarose (Sigma) for $4 \mathrm{~h}$ at $4^{\circ} \mathrm{C}$. The beads were washed eight times with buffer containing $50 \mathrm{mM}$ Tris- $\mathrm{HCl}$ ( $\mathrm{pH} 7.5$ ), $150 \mathrm{mM} \mathrm{NaCl}$, and $0.05 \% \mathrm{NP}-40$. The immunoprecipitated materials were eluted with SDS sample buffer and subjected to immunoblot analysis.

\section{RNA-binding analysis and immunoprecipitation of RNA-protein complex}

RNA binding UV cross-linking assays and immunoprecipitation of RNA-protein complex were performed as described (Gherzi et al. 2004).

\section{Mass spectrometry and LC-MS/MS analysis}

GST-BRF1 F2 and F3 fragments were phosphorylated by MK2 in vitro and subjected to in-solution digestion followed by LC-MS/ MS analysis. Phosphorylated samples were adjusted to $2 \mathrm{M}$ urea/ thiourea, reduced, alkylated, and digested with sequenced-grade modified trypsin. If required, tryptic peptides were further digested with AspN. Peptides were concentrated and desalted on reversed-phase $\mathrm{C}_{18}$ disks and resuspended in $7 \mu \mathrm{L}$ of $1 \%$ TFA/2\% acetonitrile in water for LC-MS analysis. Liquid chromatography was performed on a 1100 nano-HPLC (Agilent) coupled to a Finnigan LTQ-Orbitrap (Thermo Electron). Peptides were separated on a $\mathrm{C}_{18}$-reversed-phase column packed with Reprosil (ReproSil-Pur $\mathrm{C}_{18}-\mathrm{AQ} 3-\mu \mathrm{m}$ resin) and directly electrosprayed in the source of a LTQ-Orbitrap using $53 \mathrm{~min}$ or 100-min gradients of $2 \%-60 \%$ acetonitrile in $0.5 \%$ acetic acid at a flow of $250 \mathrm{~nL} / \mathrm{min}$. The LTQ-Orbitrap instrument was operated in the data-dependent mode switching automatically between MS survey scans (acquired in the LTQ-Orbitrap cell) and MS/MS spectra acquisition in the linear ion trap. An inclusion list, containing all possible phosphorylated and nonphosphorylated peptides of BRF1, was used for preferential sequencing. Multistage activation was enabled in all MS/MS events to improve fragmentation spectra of phosphopeptides, and the "lock mass" option was enabled in all full scans to improve mass accuracy of precursor ions (Olsen et al. 2005). Thee raw data files were converted to the Mascot generic format and searched with the Mascot search engine (http://www.matrixscience.com) against the IPI human protein database (http://www.ebi.ac.uk) containing BRF1 fragments of interest. Carbamidomethylation was selected as a fixed modification. Oxidation of methionine, $\mathrm{N}$-acetylation of the protein, and phosphorylation of serine, threonine, and tyrosine were used as variable modifications; precursor ion mass tolerance was $10 \mathrm{ppm}$ and fragment ion mass tolerance was $0.5 \mathrm{Da}$. Mass spectrometry data were visualized and validated with MSQuant (http://msquant.sourceforge.net) and exported to Excel (Microsoft) for further analysis. The probability for phosphorylation at each potential site was calculated from the PTM scores as described previously (Olsen et al. 2006).

\section{ACKNOWLEDGMENTS}

We thank Drs. P. Mitchell for anti-RRP4 antibody, M. Kiledjian for anti-DCP2 antibody, M. Gaestel for myc-MK2EE plasmid, A.-B. Shyu for HA-CCR4 plasmid, and C. Moroni for HT1080, SlowC, and anti-BRF1 antibody, and Mr. W.-J. Lin for technical assistance and suggestions. We also thank Drs. W.-C. Lin and F.-T. Lin for sharing reagents. This work was supported by NIH grant GM68758.

Received January 2, 2008; accepted January 24, 2008.

\section{REFERENCES}

Bakheet, T., Williams, B.R., and Khabar, K.S. 2006. ARED 3.0: The large and diverse AU-rich transcriptome. Nucleic Acids Res. 34: D111-D114. doi: 10.1093/nar/gkj052.

Benjamin, D., Schmidlin, M., Min, L., Gross, B., and Moroni, C. 2006. BRF1 protein turnover and mRNA decay activity are regulated by protein kinase B at the same phosphorylation sites. Mol. Cell. Biol. 26: $9497-9507$.

Blackshear, P.J. 2002. Tristetraprolin and other $\mathrm{CCCH}$ tandem zincfinger proteins in the regulation of mRNA turnover. Biochem. Soc. Trans. 30: 945-952.

Briata, P., Forcales, S.V., Ponassi, M., Corte, G., Chen, C.Y., Karin, M., Puri, P.L., and Gherzi, R. 2005. p38-dependent phosphorylation of the mRNA decay-promoting factor KSRP controls the stability of select myogenic transcripts. Mol. Cell 20: 891-903.

Carballo, E., Lai, W.S., and Blackshear, P.J. 1998. Feedback inhibition of macrophage tumor necrosis factor- $\alpha$ production by tristetraprolin. Science 281: 1001-1005.

Chen, C.Y. and Shyu, A.B. 1995. AU-rich elements: Characterization and importance in mRNA degradation. Trends Biochem. Sci. 20: $465-470$.

Chen, C.Y., Gherzi, R., Ong, S.E., Chan, E.L., Raijmakers, R., Pruijn, G.J., Stoecklin, G., Moroni, C., Mann, M., and Karin, M. 2001. AU-binding proteins recruit the exosome to degrade AREcontaining mRNAs. Cell 107: 451-464.

Chou, C.F., Mulky, A., Maitra, S., Lin, W.J., Gherzi, R., Kappes, J., and Chen, C.Y. 2006. Tethering KSRP, a decay-promoting AU-rich element-binding protein, to mRNAs elicits mRNA decay. Mol. Cell. Biol. 26: 3695-3706.

Chrestensen, C.A., Schroeder, M.J., Shabanowitz, J., Hunt, D.F., Pelo, J.W., Worthington, M.T., and Sturgill, T.W. 2004. MAPKAP kinase 2 phosphorylates tristetraprolin on in vivo sites including Ser178, a site required for 14-3-3 binding. J. Biol. Chem. 279: 10176-10184.

Dean, J.L., Sully, G., Clark, A.R., and Saklatvala, J. 2004. The involvement of AU-rich element-binding proteins in p38 mitogen-activated protein kinase pathway-mediated mRNA stabilisation. Cell. Signal. 16: 1113-1121.

Engel, K., Schultz, H., Martin, F., Kotlyarov, A., Plath, K., Hahn, M., Heinemann, U., and Gaestel, M. 1995. Constitutive activation of mitogen-activated protein kinase-activated protein kinase 2 by mutation of phosphorylation sites and an A-helix motif. J. Biol. Chem. 270: 27213-27221.

Garcia-Mayoral, M.F., Hollingworth, D., Masino, L., Diaz-Moreno, I., Kelly, G., Gherzi, R., Chou, C.F., Chen, C.Y., and Ramos, A. 2007. The structure of the C-terminal KH domains of KSRP reveals a noncanonical motif important for mRNA degradation. Structure 15: 485-498. 
Garneau, N.L., Wilusz, J., and Wilusz, C.J. 2007. The highways and byways of mRNA decay. Nat. Rev. Mol. Cell Biol. 8: 113-126.

Gherzi, R., Lee, K.Y., Briata, P., Wegmuller, D., Moroni, C., Karin, M., and Chen, C.Y. 2004. A KH domain RNA binding protein, KSRP, promotes ARE-directed mRNA turnover by recruiting the degradation machinery. Mol. Cell 14: 571-583.

Gherzi, R., Trabucchi, M., Ponassi, M., Ruggiero, T., Corte, G., Moroni, C., Chen, C.Y., Khabar, K.S., Andersen, J.S., and Briata, P. 2006. The RNA-binding protein KSRP promotes decay of $\beta$-catenin mRNA and is inactivated by PI3K-AKT signaling. PLoS Biol. 5: e5. doi: 10.1371/journal.pbio.0050005.

Lai, W.S., Carballo, E., Strum, J.R., Kennington, E.A., Phillips, R.S., and Blackshear, P.J. 1999. Evidence that tristetraprolin binds to AU-rich elements and promotes the deadenylation and destabilization of tumor necrosis factor $\alpha$ mRNA. Mol. Cell. Biol. 19: 43114323.

Lai, W.S., Carballo, E., Thorn, J.M., Kennington, E.A., and Blackshear, P.J. 2000. Interactions of $\mathrm{CCCH}$ zinc finger proteins with mRNA. Binding of tristetraprolin-related zinc finger proteins to Au-rich elements and destabilization of mRNA. J. Biol. Chem. 275: 17827-17837.

Lasa, M., Mahtani, K.R., Finch, A., Brewer, G., Saklatvala, J., and Clark, A.R. 2000. Regulation of cyclooxygenase 2 mRNA stability by the mitogen-activated protein kinase p38 signaling cascade. Mol. Cell. Biol. 20: 4265-4274.

Lin, W.J., Duffy, A., and Chen, C.Y. 2007. Localization of AU-rich element-containing mRNA in cytoplasmic granules containing exosome subunits. J. Biol. Chem. 282: 19958-19968.

Lykke-Andersen, J. 2002. Identification of a human decapping complex associated with hUpf proteins in nonsense-mediated decay. Mol. Cell. Biol. 22: 8114-8121.

Lykke-Andersen, J. and Wagner, E. 2005. Recruitment and activation of mRNA decay enzymes by two ARE-mediated decay activation domains in the proteins TTP and BRF-1. Genes \& Dev. 19:351-361.

Manke, I.A., Nguyen, A., Lim, D., Stewart, M.Q., Elia, A.E., and Yaffe, M.B. 2005. MAPKAP kinase-2 is a cell cycle checkpoint kinase that regulates the G2/M transition and $S$ phase progression in response to UV irradiation. Mol. Cell 17: 37-48.

Mitchell, P., Petfalski, E., Shevchenko, A., Mann, M., and Tollervey, D. 1997. The exosome: A conserved eukaryotic RNA processing complex containing multiple $3^{\prime} \rightarrow 5^{\prime}$ exoribonucleases. Cell 91: 457-466.

Olsen, J.V., de Godoy, L.M., Li, G., Macek, B., Mortensen, P., Pesch, R., Makarov, A., Lange, O., Horning, S., and Mann, M. 2005. Parts per million mass accuracy on an Orbitrap mass spectrometer via lock mass injection into a C-trap. Mol. Cell. Proteomics 4: 2010-2021.

Olsen, J.V., Blagoev, B., Gnad, F., Macek, B., Kumar, C., Mortensen, P., and Mann, M. 2006. Global, in vivo, and sitespecific phosphorylation dynamics in signaling networks. Cell 127: 635-648.

Rane, M.J., Coxon, P.Y., Powell, D.W., Webster, R., Klein, J.B., Pierce, W., Ping, P., and McLeish, K.R. 2001. p38 Kinasedependent MAPKAPK-2 activation functions as 3-phosphoinositide-dependent kinase-2 for Akt in human neutrophils. J. Biol. Chem. 276: 3517-3523.
Ridley, S.H., Dean, J.L., Sarsfield, S.J., Brook, M., Clark, A.R., and Saklatvala, J. 1998. A p38 MAP kinase inhibitor regulates stability of interleukin-1-induced cyclooxygenase-2 mRNA. FEBS Lett. 439: $75-80$.

Sarkar, B., Xi, Q., He, C., and Schneider, R.J. 2003. Selective degradation of AU-rich mRNAs promoted by the p37 AUF1 protein isoform. Mol. Cell. Biol. 23: 6685-6693.

Schmidlin, M., Lu, M., Leuenberger, S.A., Stoecklin, G., Mallaun, M., Gross, B., Gherzi, R., Hess, D., Hemmings, B.A., and Moroni, C. 2004. The ARE-dependent mRNA-destabilizing activity of BRF1 is regulated by protein kinase B. EMBO J. 23: 4760-4769.

Stoecklin, G., Colombi, M., Raineri, I., Leuenberger, S., Mallaun, M., Schmidlin, M., Gross, B., Lu, M., Kitamura, T., and Moroni, C. 2002. Functional cloning of BRF1, a regulator of ARE-dependent mRNA turnover. EMBO J. 21: 4709-4718.

Stoecklin, G., Stubbs, T., Kedersha, N., Wax, S., Rigby, W.F., Blackwell, T.K., and Anderson, P. 2004. MK2-induced tristetraprolin:14-3-3 complexes prevent stress granule association and ARE-mRNA decay. EMBO J. 23: 1313-1324.

Stokoe, D., Caudwell, B., Cohen, P.T., and Cohen, P. 1993. The substrate specificity and structure of mitogen-activated protein (MAP) kinase-activated protein kinase-2. Biochem. J. 296: 843849.

Wang, Z., Jiao, X., Carr-Schmid, A., and Kiledjian, M. 2002. The hDcp2 protein is a mammalian mRNA decapping enzyme. Proc. Natl. Acad. Sci. 99: 12663-12668.

Wilusz, C.J. and Wilusz, J. 2004. Bringing the role of mRNA decay in the control of gene expression into focus. Trends Genet. 20: 491-497.

Wilusz, C.J., Wormington, M., and Peltz, S.W. 2001. The cap-totail guide to mRNA turnover. Nat. Rev. Mol. Cell Biol. 2: $237-$ 246.

Winzen, R., Kracht, M., Ritter, B., Wilhelm, A., Chen, C.Y., Shyu, A.B., Muller, M., Gaestel, M., Resch, K., and Holtmann, H. 1999. The p38 MAP kinase pathway signals for cytokine-induced mRNA stabilization via MAP kinase-activated protein kinase 2 and an AU-rich region-targeted mechanism. EMBO J. 18: 4969-4980.

Wu, R., Kausar, H., Johnson, P., Montoya-Durango, D.E., Merchant, M., and Rane, M.J. 2007. Hsp27 regulates Akt activation and polymorphonuclear leukocyte apoptosis by scaffolding MK2 to Akt signal complex. J. Biol. Chem. 282: 21598-21608.

Yamashita, A., Chang, T.C., Yamashita, Y., Zhu, W., Zhong, Z., Chen, C.Y., and Shyu, A.B. 2005. Concerted action of poly(A) nucleases and decapping enzyme in mammalian mRNA turnover. Nat. Struct. Mol. Biol. 12: 1054-1063.

Zhang, W., Wagner, B.J., Ehrenman, K., Schaefer, A.W. DeMaria, C.T., Crater, D., DeHaven, K., Long, L., and Brewer, G. 1993. Purification, characterization, and cDNA cloning of an AU-rich element RNA-binding protein, AUF1. Mol. Cell. Biol. 13: 7652-7665.

Zheng, C., Lin, Z., Zhao, Z.J., Yang, Y., Niu, H., and Shen, X. 2006. MAPK-activated protein kinase-2 (MK2)-mediated formation and phosphorylation-regulated dissociation of the signal complex consisting of p38, MK2, Akt, and Hsp27. J. Biol. Chem. 281: 37215-37226. 

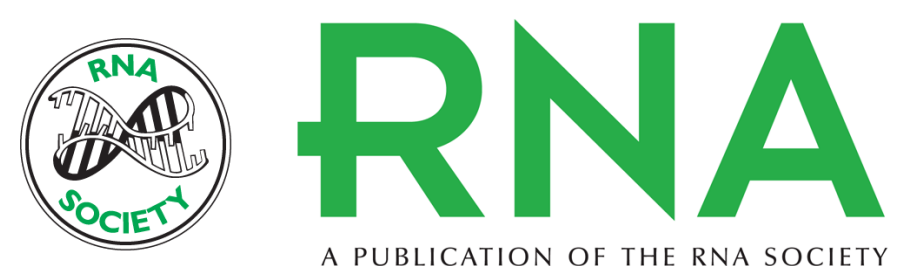

A PUBLICATION OF THE RNA SOCIETY

\section{The AU-rich element mRNA decay-promoting activity of BRF1 is regulated by mitogen-activated protein kinase-activated protein kinase 2}

Sushmit Maitra, Chu-Fang Chou, Christian A. Luber, et al.

RNA 2008 14: 950-959

References This article cites 40 articles, 23 of which can be accessed free at:

http://rnajournal.cshlp.org/content/14/5/950.full.html\#ref-list-1

License

Email Alerting Receive free email alerts when new articles cite this article - sign up in the box at the

Service top right corner of the article or click here.

To subscribe to $R N A$ go to:

http://rnajournal.cshlp.org/subscriptions 